\title{
Efficacy of Injectable Collagenase in the Treatment of Dupuytren's Contracture in Comparison to Partial Fasciectomy
}

\author{
MOHAMED A. EL-ROUBY, M.D.; AHMED ABD EL-SALAM, M.D.; AHMED GAD, M.D. and \\ KHALED RIZQ, M.D. \\ The Department of Plastic Reconstructive \& Maxillofacial Surgery, Faculty of Medicine, Ain Shams University, Cairo, Egypt
}

\begin{abstract}
Background: Dupuytren's disease as a benign fibroproliferative disease with an abnormal slowly progressive thickening and shorting of the palmar aponeurosis leads to severe functional limitations in the finger movements particularly of the Metacarpophalangeal (MCP) joints and/or the Proximal Interphalangeal (PIP) joints. The authors aimed to evaluate the role of injectable collagenase $(\mathrm{CCH})$ in the treatment of Dupuytren's contracture in comparison to surgical treatment.

Material and Methods: This study included 15 patients (33 rays), they were divided into two groups, Group A: 26 rays underwent open fasciectomy. (10 patients) and Group B: 7 rays were treated by collagenase injection. ( 5 patients). Exclusion criteria for group B were contraindications of injection of $\mathrm{CCH}$. The primary efficacy variable was clinical success, contracture correction to within $5^{\circ}$ of normal (normal $=0^{\circ}$ ) by using goniometry. Additional efficacy variables included the time and number of injections required to achieve success in the primary joint. Recurrence rate and adverse effects were recorded.

Results: Initial clinical experience was recorded of 5 patients ( 7 rays) (mean age 57 years) and compared to previously surgically managed 10 patients (26 rays) (mean age 59 years). Of all population, $51 \%$ for little, $47 \%$ for ring, $1 \%$ for middle and $1 \%$ for index fingers. The mean of the preintervention fixed flexion contracture in the MCP joint was $39^{\circ}$ and improved to one-year postintervention $14^{\circ}$, and in the PIP joint $47^{\circ}$ to $19^{\circ}$. In group B one ray with no improvement at all and recurrence in one ray. Partial stretching was achieved in one ray. No serious complications were observed after injections. In cases of group A (26 rays) that was treated by partial fasciectomy, recurrence occurred in 6 rays and nerve injuries as nerve division and neuropraxia occurred in 2 rays.

Conclusion: The treatment of Dupuytren's disease with injectable collagenase is safe and effective. However, the financial aspects should be considered especially in developing countries.
\end{abstract}

Key Words: Dupuytren finger collagenase Fasciectomy.

\section{INTRODUCTION}

Dupuytren's disease (MD) is a benign fibroproliferative disorder, named after Baron Guillaume Dupuytren's (1777-1835) [1,2], with an abnormal slowly progressive thickening and shorting of the palmar aponeurosis. The etiology of this disease remains unclear, so far, no cure is possible. It particularly affects the Metacarpophalangeal (MCP) joints and/or the proximal interphalangeal (PIP) joints. It usually affects the ring and little fingers [2].

The management of the Dupuytren's disease can be done with different modalities. The aim of all previously described therapeutic procedures is to restore normal hand function. Although surgical procedures (partial fasciectomy and fasciotomy) are still the standard, the collagenase Clostridium Histolyticum $(\mathrm{CCH})$ has been approved as safe and effective treatment option for several years. This enzymatic substance can cleave peptide bonds and degrade collagens (all human collagen types). It has the advantages of the treatment of older patients with co-morbidities, an earlier return to work, eliminate the risks of surgery [3-11].

However, it should also be noted that the results of collagenase treatment of isolated MCP contractures are significantly better than isolated PIP contractures or combined MCP/PIP contractures. Also, repeated injections of collagenase appear to be safe and effective despite the patient's antibody production because of repeated exposure. In a case report from a patient who received 15 injections over 4 years, it was found that the effect was still good in the MCP joint but slightly decreased in the PIP [12-15].

In Egypt, the commercially available collagenases (Xiapex ${ }^{\circledR}, 0.9 \mathrm{mg}$ vial, Swedish Orphan Biovitrum Ltd., Sweden or Xiaflex 0.9mg vial, Endo International plc) are a mixture, which is comprised mostly of interstitial collagen types I and III [16,17]. 
The most common complications of this nonsurgical technique are swelling, effusion, hematoma or pain. Most of these adverse effects are mild or moderate and self-limiting within an average of 10 days. Regarding the financial aspects of collagenase treatment, another disadvantage of this procedure is the higher cost that could be 200 up to 400 USD per vial [18-20].

In a review by Chen et al., compared the recurrence rates of surgical versus Non-surgical procedures showed. It was significantly higher in needle aponeurotomy than in open partial fasciectomy, and in open partial fasciectomy also appeared to be significantly higher than in $\mathrm{CCH}$ injection [18].

In 2013, the safety and efficacy of CCH injections were explored again in two studies. A total of 879 joints in 587 patients at 34 institutions were treated in the study. The treatment at an early stage is more beneficial. In $57 \%$ of the patients treatment was successful ( $0-5^{\circ}$ extension within 30 days), with more improvement in MCP than PIP joints (70 vs. 37\%) [5]. The recurrence rate was comparable to standard surgical procedures [21].

For the treatment of Dupuytren's related disease as Peyronie's disease, the use of $\mathrm{CCH}$ is both effective and safe by injection of $\mathrm{CCH}$ directly into the tunica albuginea [22].

In this study, we aimed to evaluate the efficacy of injectable collagenase (Xiapex ${ }^{\circledR}, 0.9 \mathrm{mg}$ vial, Pfizer Limited, Kent, UK) in the treatment of Dupuytren's contracture in comparison to traditional partial fasciectomy.

\section{PATIENTS AND METHODS}

This study included 15 patients (33 rays), (4 patients with 1 ray, 6 patients with 2 rays 3 patients with 3 rays, 2 patients with 2 rays) with flexion deformities of the MCP and/or PIP joints of $20^{\circ}$ or greater. Their age ranged from 40 to 65 years. They were divided into two groups:

- Group A: 26 rays underwent open fasciectomy. (10 patients).

- Group B: 7 rays were treated by collagenase injection. (5 patients).

Exclusion criteria for group B were contraindications of injection of $\mathrm{CCH}$ as pregnancy, hypersensitivity to collagenase or patients uses anticoagulant.

All the patients' results were recorded at 3 rd, $6^{\text {th }}$ and $18^{\text {th }}$ moths. The primary efficacy variable was clinical success, contracture correction to within $5^{\circ}$ of normal (normal $=0^{\circ}$ ) by using goniometry. Additional efficacy variables included the time and number of injections required to achieve success in the primary joint. Recurrence rate and adverse effects were recorded. Common side effects include local swelling, redness, or pain at the site of puncture was also recorded.

\section{Injection procedure:}

After injection of local anesthetic (Lidocaine $1: 200000), 0.3 \mathrm{mg}$ per injection of Xiapex ${ }^{\circledR}(0.9 \mathrm{mg}$ vial, Pfizer Limited, Kent, UK) [23] was injected into the palpable cord. After 24-72 hours, we perform finger extension procedure to facilitate cord disruption for approximately 10-20 seconds. If the first attempt to extend the finger is unsuccessful, a second and a third attempt can be made at intervals of 5-10 minutes each without increasing the force. The patients were instructed to use splint for 10 days with active and passive extension exercises. Injection was repeated after four weeks, if the MCP or PIP contracture remains, up to a maximum of 3 injections in the same strand.

\section{RESULTS}

Initial clinical experience was recorded of 5 patients ( 7 rays) (4 males, 1 female, mean age 57 [range 40-61] years) Figs. $(1,2)$ and compared to previously surgically managed 10 patients (26 rays) (7 males, 3 females mean age 59 [range 42-65] years) Figs. $(3,4)$ (Table 1). Of all population, $51 \%$ were treated for little fingers, $47 \%$ for ring fingers, $1 \%$ for middle fingers and $1 \%$ for index fingers.

A successful strand solution was defined as achieving $0-5^{\circ}$ extension of the affected joint. This was achieved in all group B cases except one ray with no improvement at all and recurrence in one ray. Partial stretching was achieved in one ray.

The mean of the pre-intervention fixed flexion contracture in the MCP joint was $39^{\circ}$ and improved to one-year postintervention $14^{\circ}$, and in the PIP joint $47^{\circ}$ to $19^{\circ}$ Fig. (2).

No serious complications (tendon/ligament ruptures, nerve lesions or hypersensitivity reactions) were observed after injections. In cases of group A (26 rays) that was treated by partial fasciectomy, recurrence occurred in 6 rays and nerve injuries as nerve division and neuropraxia occurred in 2 rays (Table 2 ). 


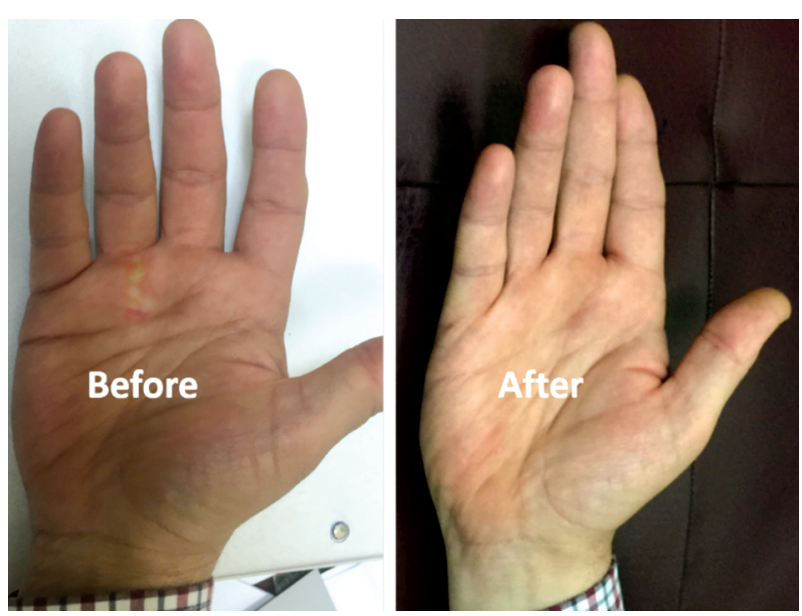

Fig. (1): Pre and 6 months after injection of right ring finger MCP joint (Group B).
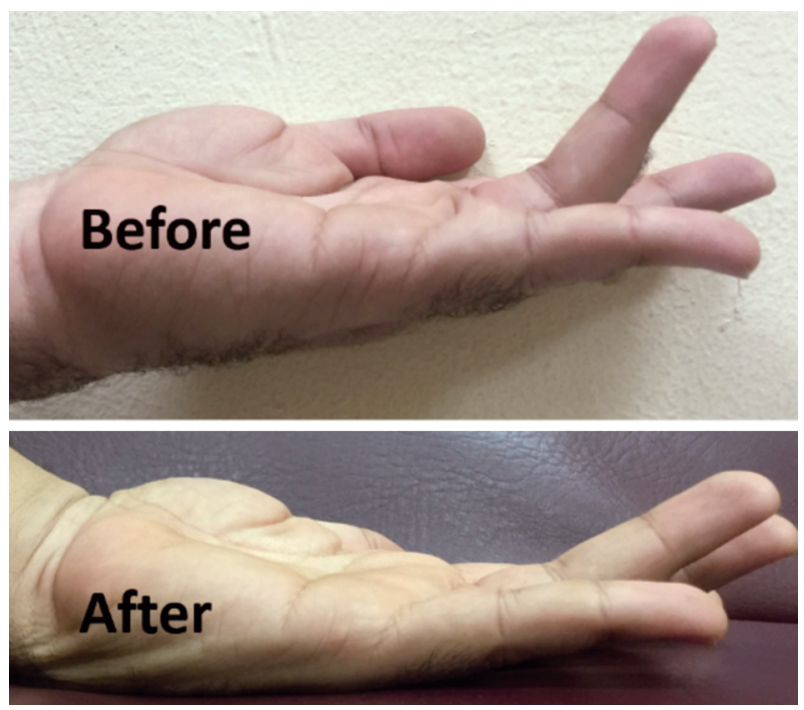

Fig. (2): Lateral view show improvement of flexion deformity of left PIP joint of ring finger from $35^{\circ}$ to $15^{\circ}($ Group B).
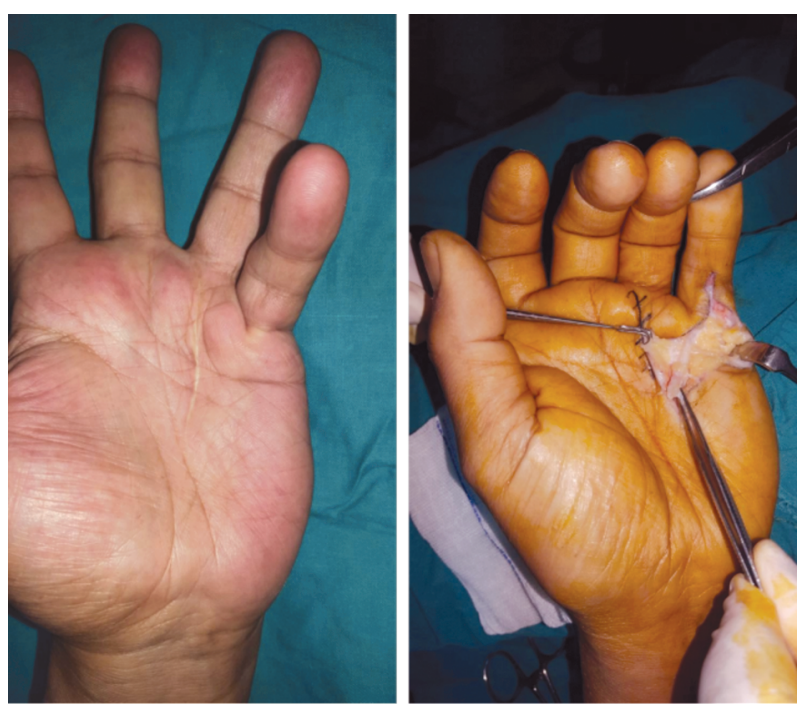

Fig. (3): Pre-operative and intraoperative of partial fasciectomy (Group A).

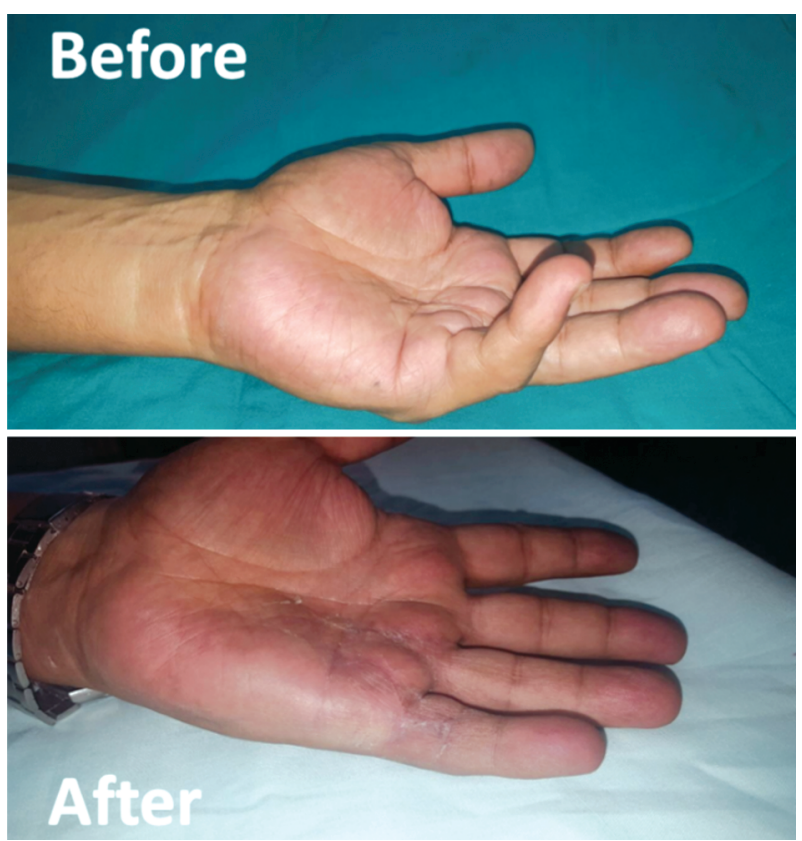

Fig. (4): Lateral view show improvement of flexion deformity of left MCP joint of little finger from $49^{\circ}$ to $9^{\circ}$.

Table (1): Number of treated rays in each group.

Group A: 26 rays underwent open fasciectomy. (10 patients):

- 1 patient $\rightarrow 1$ ray

- 4 patients $\rightarrow 2$ rays

- 3 patients $\rightarrow 3$ rays

- 2 patients $\rightarrow 2$ rays

Group B: 7 rays were treated by collagenase injection. (5 patients):

- 3 patients $\rightarrow 1$ ray

- 2 patient $\rightarrow 2$ rays

Table (2): Recorded complication in both groups.

\begin{tabular}{ll}
\hline $\begin{array}{c}\text { Open Fasciectomy } \\
(26 \text { rays })\end{array}$ & $\begin{array}{c}\text { Collagenase injection } \\
\text { (7 rays) }\end{array}$ \\
\hline Recurrence 6/26 & Recurrence $1 / 7$ \\
Nerve division $1 / 26$ & Failure of treatment $1 / 7$ \\
Neuropraxia 1/26 & Partial stretch $1 / 7$ \\
Infection 0 & Nerve injury 0 \\
Skin sloughing 0 & Hypersensitivity 0 \\
\hline
\end{tabular}

\section{DISCUSSION}

Our experience has shown that the use of injectable collagenase is an effective and welltolerated alternative to surgical treatment.

The patient must, however, be informed about the frequent occurrence of undesired side effects (especially cost and hypersensitivity) as well as recurrence rate and ineffectiveness of the treatment that may need surgical intervention. 
As regard the undesirable side effects in group $\mathrm{B}$, they were minimally and self-limiting like swelling, bleeding, pain, redness in the puncture site. No nerve lesions were detected.

In elderly patients with multiple co-morbidities, who should avoid anesthesia or postoperative wound healing problems, treatment with $\mathrm{CCH}$ is a very good alternative.

The effectiveness and patient satisfaction of collagenase treatment versus partial fasciectomy are comparable; in view of the earlier return to work and surgical complications, collagenase injection has the advantage.

However, the question of the significance in relation to partial fasciectomy cannot yet be answered conclusively. The published recurrence rate shows considerable differences. The method will have to be measured for the long-term recurrence rate and complications.

\section{Conclusion:}

The treatment of Dupuytren's disease with injectable collagenase has been proven safe and effective, as confirmed by recent studies. However, the financial aspects should be considered especially in developing countries.

\section{Conflict of interest:}

There is no conflict of interest.

\section{REFERENCES}

1- Shaw Jr. R.B., Chong A.K., Zhang A., Hentz V.R. and Chang J.: Dupuytren's disease: History, diagnosis, and treatment. J. Plastic Reconstructive Surgery, 120: 44e$54 \mathrm{e}, 2007$.

2- Townley W., Baker R., Sheppard N. and Grobbelaar A.: Dupuytren's contracture unfolded. J. B.M.J.: British Medical Journal, 332: 397, 2006.

3- Watt A.J., Curtin C.M. and Hentz V.R.: Collagenase injection as nonsurgical treatment of Dupuytren's disease: 8 -year follow-up. J. The Journal of hand surgery, 35: 534539. e531, 2010.

4- Gelbard M.K., James K., Riach P. and Dorey F.: Collagenase versus placebo in the treatment of Peyronie's disease: A double-blind study. J. The Journal of Urology, 149: 56-58, 1993.

5- Nydick J.A., Olliff B.W., Garcia M.J., Hess A.V. and Stone J.D.: A comparison of percutaneous needle fasciotomy and collagenase injection for Dupuytren disease. J. The Journal of Hand Surgery, 38: 2377-2380, 2013.

6- Peimer C.A., Blazar P., Coleman S., Kaplan F.T.D., Smith T. and Lindau T.: Dupuytren contracture recurrence following treatment with collagenase clostridium histolyticum (CORDLESS [Collagenase Option for Reduction of Dupuytren Long-Term Evaluation of Safety Study]): 5-year data. J. The Journal of Hand Surgery, 40: 1597-1605, 2015.

7- Skirven T.M., Bachoura A., Jacoby S.M., Culp R.W. and Osterman A.L.: The effect of a therapy protocol for increasing correction of severely contracted proximal interphalangeal joints caused by Dupuytren disease and treated with collagenase injection. J. The Journal of Hand Surgery, 38: 684-689, 2013.

8- Coleman S., Gilpin D., Kaplan F.T.D., et al.: Efficacy and safety of concurrent collagenase clostridium histolyticum injections for multiple Dupuytren contractures. J. The Journal of Hand Surgery, 39: 57-64, 2014.

9- Hovius S.E., Kan H.J., Smit X., Selles R.W., Cardoso E. and Khouri R.K.: Extensive percutaneous aponeurotomy and lipografting: A new treatment for Dupuytren disease. J. Plastic Reconstructive Surgery, 128: 221-228, 2011.

10- Van Rijssen A.L., Ter Linden H. and Werker P.M.: Fiveyear results of a randomized clinical trial on treatment in Dupuytren's disease: Percutaneous needle fasciotomy versus limited fasciectomy. J. Plastic Reconstructive Surgery, 129: 469-477, 2012.

11- Scherman P., Jenmalm P. and Dahlin L.: One-year results of needle fasciotomy and collagenase injection in treatment of Dupuytren's contracture: A two-centre prospective randomized clinical trial. J. Journal of Hand Surgery, 41: 577-582, 2016.

12- Desai S.S. and Hentz V.R.: Collagenase clostridium histolyticum for Dupuytren's contracture. J. Expert Opinion on Biological Therapy, 10: 1395-1404, 2010.

13- Warwick D., Arner M., Pajardi G., et al.: Collagenase Clostridium histolyticum in patients with Dupuytren's contracture: Results from POINT X, an open-label study of clinical and patient-reported outcomes. J. Journal of Hand Surgery, 40: 124-132, 2015.

14- Zhou, C., Hovius, S.E., Slijper, H.P., et al. Collagenase clostridium histolyticum versus limited fasciectomy for Dupuytren's contracture: outcomes from a multicenter propensity score matched study. J. Plastic Reconstructive Surgery, 136: 87-97, 2015.

15- Starkweather K.D., Lattuga S., Hurst L.C., et al.: Collagenase in the treatment of Dupuytren's disease: $\mathrm{AN}$ in vitro study. J. The Journal of Hand Surgery, 21: 490-495, 1996.

16- Badalamente M.A., Hurst L.C. and Hentz V.R.: Collagen as a clinical target: Nonoperative treatment of Dupuytren's disease. J. The Journal of Hand Surgery, 27: 788-798, 2002.

17- Peimer C., Wilbrand S., Gerber R., Chapman D. and Szczypa P.: Safety and tolerability of collagenase Clostridium histolyticum and fasciectomy for Dupuytren's contracture. J. Journal of Hand Surgery, 40: 141-9, 2015.

18- Chen N.C., Shauver M.J. and Chung K.C.: Costeffectiveness of open partial fasciectomy, needle aponeurotomy, and collagenase injection for Dupuytren contracture. J. The Journal of Hand Surgery, 36: 1826-1834. e1832, 2011.

19- Atroshi I., Strandberg E., Lauritzson A., Ahlgren E. and Waldén M.: Costs for collagenase injections compared with fasciectomy in the treatment of Dupuytren's contrac- 
ture: A retrospective cohort study. J. B.M.J. open, 4: e004166, 2014.

20- Cerveró R.S., Ferrando N.F. and Jornet J.P.: Use of resources and costs associated with the treatment of Dupuytren's contracture at an orthopedics and traumatology surgery department in Denia (Spain): Collagenase clostridium hystolyticum versus subtotal fasciectomy. J. B.M.C. Musculoskeletal Disorders, 14: 293, 2013.

21- Peimer C.A., Blazar P., Coleman S., et al.: Dupuytren contracture recurrence following treatment with collagenase clostridium histolyticum (CORDLESS study): 3- year data. J. The Journal of Hand Surgery, 38: 12-22, 2013.

22- Lipshultz L.I., Goldstein I., Seftel A.D., et al.: Clinical efficacy of collagenase Clostridium histolyticum in the treatment of Peyronie's disease by subgroup: Results from two large, double-blind, randomized, placebo-controlled, phase III studies, 116: 650-656, 2015.

23- Povlsen B. and Povlsen S.D.J.H.S.: What is the better treatment for single digit dupuytren's contracture: Surgical release or collagenase clostridium histolyticum (Xiapex) injection? 19: 389-392, 2014. 